This is a postprint version of the following published document:

González-Arribas, D., Hentzen, D., Sanjurjo-Rivo, M., Soler, M. \& Kamgarpour, M. (2017). Optimal Aircraft Trajectory Planning in the Presence of Stochastic Convective Weather Cells. 17th AIAA Aviation Technology, Integration, and Operations Conference, AIAA AVIATION Forum, Denver, Colorado. AIAA 2017-3431, pp. 1-13.

DOI: $10.2514 / 6.2017-3431$

(C) The American Institute of Aeronautics and Astronautics, 2017 


\title{
Optimal Aircraft Trajectory Planning in the Presence of Stochastic Convective Weather Cells
}

\author{
Daniel González-Arribas, ${ }^{*}$ Daniel Hentzen, ${ }^{\dagger}$ Manuel Sanjurjo-Rivo, ${ }^{\ddagger}$ \\ Manuel Soler, ${ }^{\ddagger}$ and Maryam Kamgarpour ${ }^{\S}$ \\ Automatic Control Laboratory, ETH Zürich, Switzerland \\ Department of Bioengineering and Aerospace Engineering, Universidad Carlos III de Madrid, Leganés, Spain
}

\begin{abstract}
The Air Traffic Management system is heavily influenced by meteorological uncertainty, and convective weather cells represent one of the most relevant uncertain meteorological phenomena. They are weather hazards that must be avoided through tactical trajectory modifications. As a consequence of the existence in uncertainty in meteorological forecasts and nowcasts, it is important to consider the convective weather cells to be avoided as a stochastic, time-dependent process. In this paper we present a comparative analysis of two methodologies for handling stochastic storms in trajectory planning: one based on stochastic reachability and a second one, based on robust optimal control. In the former, the thunderstorm avoidance problem is modelled as a stochastic reach-avoid problem, consid-ering the motion of the aircraft as a discrete-time stochastic system and the weather haz-ards as random set-valued obstacles. Dynamic programming is used to compute a Markov feedback policy that maximizes the probability of reaching the target before entering the unsafe set, i.e., the hazardous weather zones. For the latter, the stochastic dynamics of the storms are modeled in continuous time. We implement an optimal control formulation that allows different possible realizations of the stochastic process to be considered.

The resulting problem is then transcribed to a nonlinear programming (NLP) problem through the use of direct numerical methods. A benchmark case study is presented, in which the effectiveness of the two proposed approaches are analyzed.
\end{abstract}

\section{Introduction}

Meteorological uncertainty represents one of the most important phenomena affecting Air Traffic Management (ATM) performance. In particular, the inherent uncertainty in convective weather cells (commonly referred to as storms) results in a major safety hazard to aircraft, potentially leading to traffic disruptions, delays, ${ }^{1}$ and, in turn, inefficiencies and $\operatorname{costs}^{2}$ for both airlines and passengers. Convective weather cells are usually avoided by pilots and air traffic controllers through route modifications, deviating from the previously filed flight plan. However, without an automated rerouting system that considers the future likely evolution of the weather hazards and the performance of the aircraft, the new flight plans chosen by the pilot or the air traffic controller may be inefficient or not safe enough. They may also involve high workloads, which has consequences in terms of safety and ATM capacity.

In order to improve ATM safety and reduce delays and fuel consumption, it is thus paramount to cope with convective weather cells and their associated uncertainty. According to the future Trajectory Based Operations concept ${ }^{3}$ to be implemented through SESAR in Europe, aircraft will agree to fly a so-called reference business trajectory (calculated before departure), being able to revise, modify, and update it upon

\footnotetext{
${ }^{*}$ PhD Candidate, Department of Bioengineering and Aerospace Engineering (dangonza@ing.uc3m.es)

${ }^{\dagger}$ MSc Student, Automatic Control Laboratory, ETH Zürich

$¥$ Assistant Professor, Department of Bioengineering and Aerospace Engineering, Universidad Carlos III de Madrid

§Professor, Automatic Control Laboratory, ETH Zürich
} 
certain circumstances (resulting thus in a revised reference business trajectory). A vital aspect in this trajectory updating process is convective weather cell avoidance, in which not only the trajectory evolution itself but also the convective weather cell to be avoided are modeled as time-dependent, stochastic processes. The synthesis of trajectories that are robust to such uncertainty is especially relevant for operations that demand the highest possible levels of safety, e.g. aircraft traffic management and path planning through hazardous weather regions. As a consequence, developing algorithms to perform this task automatically and proposing the required modifications while taking advantage of the latest meteorological solutions will lead to more efficient and safe operations in bad weather conditions.

There has been some attention in the ATM research community to develop and introduce such methods and tools. In an analysis ${ }^{4}$ of operational experience since the implementation of dynamic weather routes in American Airlines, the authors found savings of an average 6.7 minutes per flight and a potential reduction in sector congestion of $20 \%$. These results were attained with a basic path planning algorithm on top of an operational tool. A different tool, DIVMET, ${ }^{5-7}$ uses beam-scanning path planning algorithms in order to route aircraft around weather hazards. In related work, uncertainty bounds for storms that are identified with a radar-based nowcast system have been derived. ${ }^{8}$

Our main contribution to the literature is the consideration of uncertainty in the evolution of the storms as well as the employment of 4D trajectory capabilities, in line with the Trajectory-Based Operations model employed in incoming ATM paradigms. From the mathematical point-of-view, this problem presents significant challenges, due to the nonlinear dynamics and nonconvex constraints generated by modeling the weather hazards as mathematical obstacles.

In this work, we will implement and evaluate two algorithms in order to automatically propose safe trajectory modifications in the presence of stochastically-evolving convective weather cells. Given an initial flight plan and trajectory that is blocked by convective weather cells, we will seek to obtain a trajectory modification that avoids the weather hazards with either maximum safety levels (stochastic reach-avoid framework) or with minimal cost and time deviation (optimal control framework).

A first approach we propose to maximize both reachability and safety is to formulate and solve a stochastic reach-avoid problem. The objective is to maximize the probability of reaching a target set while avoiding a stochastic obstacle set that represents the convective weather cells, given an initial condition and a fixed time horizon. The obstacle set is modeled as a time-indexed sequence of random closed sets. ${ }^{9}$ The stochastic reach-avoid problem is cast as a discrete time, finite horizon stochastic optimal control problem ${ }^{10}$ with a sum-multiplicative cost-to-go function. The optimal Markov control policy is computed using dynamic programming. ${ }^{11}$

The second approach that we will consider is based on optimal control techniques. We model the stochastic evolution of the convective weather cells in continuous time in a manner that is tractable by numerical algorithms. We then discretize the uncertainty into multiple scenarios in order to apply an optimal control formulation that is adapted for the consideration of a discrete set of possible realizations. ${ }^{12}$ We finally discretize the resulting optimal control problem into a nonlinear optimization problem through the use of direct methods.

We set up a benchmark problem to compare both methods. These results will serve as a basis for our future work, where we plan to test the algorithms on complex scenarios based on real storm data.

This paper is structured as follows: Section II outlines the reach-avoid framework while Section III describes the optimal control methodology. Section IV presents a benchmark problem and the computational parameters while Section V shows and discusses the results. Finally, we draw some conclusions and discuss potential future work in Section VI. 


\section{Reach-Avoid Methodology}

The first methodology that we consider models the thunderstorm avoidance problem in discrete time as a stochastic reach-avoid problem with random set-valued obstacles. It is solved with stochastic dynamic programming. This problem has been well-studied in the literature. ${ }^{9,13}$ We will consider the motion of the aircraft as a discrete-time stochastic system. The stochasticity stems from the uncertainty in the trajectory evolution, due to potential wind and process noise, and in the convective weather cell characterization. Dynamic programming will be used to compute a Markov feedback policy that maximizes the probability of reaching the target before entering the unsafe set, that is, the hazardous weather zones.

\section{A. Discrete Time Stochastic Dynamical System}

Let $X \subseteq \mathbb{R}^{n}$ and $U \subset \mathbb{R}^{m}$ be Borel sets representing the state and input spaces, respectively. Let $\mathcal{B}(S)$ denote the set of Borel subsets of a given space $S$. A stochastic dynamical system is described by:

$$
\mathbf{x}_{t+1} \sim \tau\left(B \mid \mathbf{x}_{t}, \mathbf{u}_{t}\right), \quad t=0,1, \ldots, N
$$

where $\mathbf{x}_{t} \in X$ and $\mathbf{u}_{t} \in U$ are the state and input at time $t$, and $B \in \mathcal{B}(X)$. The function $\tau: \mathcal{B}(X) \times X \times U \rightarrow$ $[0,1]$ is a Borel measurable stochastic kernel, ${ }^{14}$ assigning to each $\mathbf{x}_{t} \in X$ and $\mathbf{u}_{t} \in U$ a probability measure on the Borel space $\mathcal{B}(X)$. The state $\mathbf{x}_{t+1}$ is thus sampled from the probability distribution $\tau\left(\cdot \mid \mathbf{x}_{t}, \mathbf{u}_{t}\right)$.

\section{B. Random Obstacle Sets}

The obstacle set is modeled as a random closed set $\Xi$. Its movement is described by a stochastic set-valued Markov process. ${ }^{9}$ Let $p_{\Xi}(x)=P\{x \in \Xi\}$ denote the so-called covering function, ${ }^{14}$ mapping to each $x \in X$ a probability measure of being in the obstacle set.

To alleviate the complexity of characterizing the stochastic set process and computing the associated covering function, we assume that the covering function is described by a finite dimensional stochastic parameter (in our example, the center and the orientation of the storm). The covering function can then be computed either analytically (depending on the distribution of the parameter) or via Monte Carlo simulation. In subsection $\mathrm{D}$, we model the weather hazards with this formulation.

\section{Aircraft Dynamics}

We model the aircraft as a point-mass unicycle flying at constant airspeed (a model also known as a Dubins vehicle) with three modes of operation: straight flight, right turn and left turn. The discrete time dynamics of the aircraft are given by

$$
\begin{aligned}
& x_{k+1}=x_{k}+v \cos \left(\chi_{k}\right) \Delta t+w_{k}^{1} \\
& y_{k+1}=y_{k}+v \sin \left(\chi_{k}\right) \Delta t+w_{k}^{2} \\
& \chi_{k+1}=\chi_{k}+u_{k} \Delta t+w_{k}^{3}
\end{aligned}
$$

where $[x y] \in X \times Y \subset \mathbb{R}^{2}$ is the two-dimensional position of the aircraft $\chi \in[-\pi, \pi]$ is the heading of the aircraft (measured from the geographic North in clockwise fashion), $u$ is the turning rate of the aircraft and unique control variable, $w=\left[w^{1}, w^{2}, w^{3}\right]^{T} \sim \mathcal{N}\left(0, \Sigma_{w}\right)$ is the stochastic process disturbance of the system due to wind and model uncertainties and $\Delta t$ is the sampling time. In line with the three modes of operation of the aircraft, it takes one of three possible values: $u \in\{-\Omega, 0, \Omega\}$.

\section{Stochastic Storm Characterization}

The characterization of moving obstacles that the aircraft must avoid is a challenging problem. Predicted storm cell parameters such as position, speed and size, as well as meteorological measurements such as Vertically Integrated Liquid (VIL) are readily available in regular time steps and over the horizon of an hour or more. However, the associated uncertainty should be accounted for, in particular for longer forecast horizons. To address the resulting probabilistic nature of the no-fly regions, they can be modeled as random set-valued processes. ${ }^{9}$ 
To enable a parameterized process and thus alleviate complexity as described in Section B, the no-fly regions can be represented by enclosing them with minimum-volume ellipsoids. For further simplification, we assume constant flight levels and thus two-dimensional bounding ellipses.

Let $L_{k}$ be the number of obstacles in the forecast data at time $k$ and let $q=[x y]^{T} \in \mathbb{R}^{2}$ be the position vector. The elliptical obstacles are given by the set $\left\{\varepsilon_{k}^{l}\right\}$ where $l=1,2, \ldots, L_{k}$. Each ellipse $\varepsilon_{k}^{l}$ is parameterized by its center $m_{k}^{l} \in \mathbb{R}^{2}$ and its positive definite eccentricity matrix $M_{k}^{l}$ :

$$
q \in \varepsilon_{k}^{l}\left(m_{k}^{l}, M_{k}^{l}\right) \Longleftrightarrow\left(q-m_{k}^{l}\right)^{T} M_{k}^{l}\left(q-m_{k}^{l}\right) \leq 1 .
$$

To further simplify the notation, we consider only one hazardous weather region (i.e. $l=1$ ).

We can now model the obstacle as a random closed set, where $m_{k}$ and $M_{k}$ are provided in the forecast data, resulting in a set-valued Markov process. Let $\xi$ be a random variable denoting the true realization of the ellipse center. Its dynamics are given by

$$
\xi_{k+1}=\xi_{k}+\lambda_{k}+\eta_{k}
$$

where $\lambda_{k}=m_{k+1}-m_{k}$ and $\eta_{k} \sim \mathcal{N}(0, \Sigma)$. The motion of the thunderstorm is therefore composed by a deterministic term, drawn from the forecasted evolution of the center $m_{k}$, and a stochastic term $\eta_{k}$.

The variation in the ellipse eccentricity $M_{k}$ is approximated as

$$
C_{k}=R\left(\theta_{k}\right)^{T} M_{k} R\left(\theta_{k}\right),
$$

where $R(\cdot)$ is a rotation matrix with a rotation angle $\theta_{k} \sim \mathcal{U}(-\alpha, \alpha)$, and $\mathcal{U}(-\alpha, \alpha)$ is the uniform distribution on $[-\alpha, \alpha]$. The parameters $\Sigma$ and $\alpha$ can be determined from forecast data and associated statistics. ${ }^{15}$

The covering function for the random closed sets, that is, given a position $q_{k}$, the probability of being in $\left.\Xi_{k}=\varepsilon_{k}\left(\xi_{k}, C_{k}\right)\right)$, can be written as follows:

$$
p_{\Xi}\left(q_{k}\right)=P\left(q_{k} \in \Xi_{k}\right)=P\left\{\left(q_{k}-\xi_{k}\right)^{T} C_{k}\left(q_{k}-\xi_{k}\right) \leq 1\right\} .
$$

It can be computed using Monte Carlo methods.

\section{E. Finite Horizon Reach-Avoid}

Let $K$ be the target set and $K_{k}^{\prime}$ the safe set, with $K \subseteq K_{k}^{\prime}$ for all $k$. We aim to evaluate and maximize the probability of reaching $K$ before intersecting the obstacle set $X \backslash K_{k}^{\prime}$ within $N$ steps. While the initial obstacle set $\Xi_{0}$ is assumed known, $\Xi_{k}$ is an execution of the stochastic set-valued process defined in Section $\mathrm{D}$, for $k=1, \ldots, N$.

Let the sequence $\mu=\left\{\mu_{0}, \mu_{1}, \ldots, \mu_{N-1}\right\}$ be a Markov policy, that is $\mu_{k}: X \rightarrow U$. Let $\mathcal{M}_{m}$ denote the set of all admissible Markov policies and let $\mathbf{1}_{A}$ denote the indicator function of the set $A$. As proved in, ${ }^{9}$ the probability that the system initialized at $\mathbf{x}_{0} \in X$ with control policy $\mu \in \mathcal{M}_{m}$ and the initial obstacle set $\Xi_{0} \in \mathcal{K}$ reaches $K$ before intersecting $X \backslash K_{k}^{\prime}$ is given as:

$$
r_{\mathbf{x}_{0}, \Xi_{0}}^{\mu}(K)=E_{\mathbf{x}_{0}, \Xi_{0}}^{\mu}\left[\sum_{j=0}^{N}\left(\prod_{i=0}^{j-1} \mathbf{1}_{K_{i}^{\prime} \backslash K}\left(\mathbf{x}_{i}\right)\right) \mathbf{1}_{K}\left(\mathbf{x}_{j}\right)\right]=E_{\mathbf{x}_{0}}^{\mu}\left[\sum_{j=0}^{N}\left(\prod_{i=0}^{j-1} p_{K_{i}^{\prime} \backslash K}\left(\mathbf{x}_{i}\right)\right) \mathbf{1}_{K}\left(\mathbf{x}_{j}\right)\right] .
$$

Maximizing this probability is equivalent to computing $r^{*}\left(\mathbf{x}_{0}, \Xi_{0}\right):=\sup _{\mu \in \mathcal{M}_{m}} r^{\mu}\left(\mathbf{x}_{0}, \Xi_{0}\right)(K)$. This can be achieved by solving a stochastic optimal control problem via dynamic programming. For a fixed Markov policy $\mu \in \mathcal{M}_{m}$, we define the cost function $V_{k}^{\mu}: X \rightarrow[0,1], k=0, \ldots, N$. The total cost to be maximized is defined as:

$$
V_{0}^{\mu}\left(\mathbf{x}_{0}\right):=r_{\mathbf{x}_{0}, \Xi_{0}}^{\mu}(K)
$$


The backwards recursion needed to compute the optimal cost-to-go $V_{k}^{\mu *}$ for $k=N-1, \ldots, 0$ is as follows:

$$
V_{k}^{\mu *}=\mathbf{1}_{K}\left(\mathbf{x}_{k}\right)+p_{K_{k}^{\prime} \backslash K}\left(\mathbf{x}_{k}\right) \sup _{u_{k} \in U} \int_{X} V_{k+1}^{\mu *}\left(\mathbf{x}_{k+1}\right) \cdot \tau\left(\mathbf{x}_{k+1} \mid \mathbf{x}_{k}, \mathbf{u}_{k}\right)
$$

The recursion is initialized with $V_{N}^{\mu}\left(\mathbf{x}_{N}\right)=\mathbf{1}_{K}\left(\mathbf{x}_{N}\right)$. An optimal reach-avoid policy $\mu_{k}^{*} \in \mathcal{M}_{m}$ for $k=$ $0, \ldots, N-1$ is given by:

$$
\mu_{k}^{*}\left(\mathbf{x}_{k}\right) \in \arg \sup _{u_{k} \in U}\left\{\mathbf{1}_{K}\left(\mathbf{x}_{k}\right)+p_{K_{k}^{\prime} \backslash K}\left(\mathbf{x}_{k+1}\right) \int_{X} V_{k+1}^{\mu *}\left(\mathbf{x}_{k+1}\right) \cdot \tau\left(\mathbf{x}_{k+1} \mid \mathbf{x}_{k}, \mathbf{u}_{k}\right)\right\} .
$$

\section{Optimal Control Methodology}

The second methodology that will be considered in this study is an optimal control-based methodology. We will model the stochastic dynamics of the storms in continuous time and formulate an optimal control problem, which can then be transcribed to a nonlinear programming (NLP) problem through the use of direct numerical methods.

\section{A. Aircraft dynamics}

We consider again a point-mass model of the aircraft as in BADA, ${ }^{16,17}$ simplified for motion in a $2 \mathrm{D}$ plane ${ }^{\mathrm{a}}$ with constant airspeed. The state vector of the aircraft is given by $\mathbf{x}=\left[\begin{array}{lll}x & y & \chi\end{array}\right]^{T}$, where $x$ is the horizontal coordinate of the position, $y$ is the vertical coordinate, and $\chi$ is the heading of the aircraft, measured from the geographic North. The control is the bank angle of the aircraft: $u=\psi$. The continuous-time dynamics of the aircraft are given by the differential equations:

$$
\begin{gathered}
\dot{x}=v \sin \chi \\
\dot{y}=v \cos \chi \\
\dot{\chi}=(g / v) \tan \psi,
\end{gathered}
$$

where $g=9.8 \mathrm{~m} \cdot \mathrm{s}^{-2}$ is the gravitational acceleration. In addition, the control is limited by the constraint

$$
|\psi| \leq \pi / 4
$$

which is consistent with BADA 3 limits. It is also well below the load factor limits in BADA 4 for a constant-airspeed and constant-altitude turn.

For the purposes of this work, we don't consider the effect of the wind; however, we will note that we can use methods described in the literature ${ }^{18}$ in order to model wind and incorporate it into the problem in a computationally efficient way. In addition, the dynamics of the aircraft are considered to be deterministic as the uncertainty in them is assumed to be less important than the uncertainty in the expected position of the storm.

\section{B. Storm motion}

As in Section II, we consider an elliptical model of the storms and obstacles; however, because the optimal control problem is formulated in continuous time, there are some relevant differences that we will address within the framework of stochastic differential equations (in the sense of Itô stochastic differential equations $\left.{ }^{19}\right)$. For simplicity, let us discuss the case of a single ellipse with center $m_{t}$ at time $t$. The continuous-time equivalent of the model of the motion of the center of the storm presented in Equation (3) is a Brownian motion with drift, i.e.

$$
\xi_{t}=\xi_{0}+\tilde{\lambda} t+\tilde{\Sigma} W_{t}
$$

${ }^{a}$ We also have implemented motion in an ellipsoidal field, but considering the short distances involved in this work and for comparison purposes it is enough to consider a $2 \mathrm{D}$ plane. 
where $\xi_{t}$ is the position of the storm at moment $t, \tilde{\lambda} \in \mathbb{R}^{2}$ is the expected speed of motion of the storm (corresponding with the deterministic forecast), $\tilde{\Sigma} \in \mathbb{R}^{2 \times 2}$ is the covariance matrix of the stochastic part of the motion while $W_{t}$ is a two-dimensional, uncorrelated Wiener process. Therefore, the equivalent stochastic differential equation is:

$$
d \xi=\tilde{\lambda} d t+\tilde{\Sigma} d W
$$

Let $\Delta t$ be the step size employed in Section II. Then, if we choose:

$$
\begin{gathered}
\tilde{\lambda}=\frac{\lambda}{\Delta t} \\
\tilde{\Sigma}=\frac{1}{\Delta t} \Sigma,
\end{gathered}
$$

the discrete-time model in Equation (3) is exactly equivalent to the continuous-time model sampled at intervals of size $\Delta t$. However, this creates a new challenge for optimal control problems where the final time is free (implying a variable time-step). Not only do we need a closed-form expression for $\xi(t)$ when transcribing the optimal control problem with a direct method, but we also need it to be a differentiable function for the nonlinear programming solver to run successfully, and a Wiener process is not differentiable. In order to address this challenge, we consider the Karhunen-Loève expansion of the Wiener process for $t \in[0,1]$. A Wiener process in this interval can be represented by:

$$
W_{t}=\sqrt{2} \sum_{k=1}^{\infty} Z_{k} \frac{\sin \left(\left(k-\frac{1}{2}\right) \pi t\right)}{\left(k-\frac{1}{2}\right) \pi}
$$

where $\left\{Z_{k}\right\}$ are i.i.d. standard normals. We take a truncated sum by keeping only the first $n_{K L}$ terms, therefore approximating the Brownian motion with the sum of its higher-amplitude, lower-frequency components. A bound of the error is presented in Lemma III.1.

Lemma III.1. The variance of the amplitude of the error is $\mathcal{O}\left(1 / n_{K L}\right)$

Proof.

$$
\begin{gathered}
\operatorname{Var}\left[\sqrt{2} \sum_{k=n_{K L}+1}^{\infty} Z_{k} \frac{\sin \left(\left(k-\frac{1}{2}\right) \pi t\right)}{\left(k-\frac{1}{2}\right) \pi}\right] \leq \operatorname{Var}\left[\sqrt{2} \sum_{k=n_{K L}+1}^{\infty} Z_{k} \frac{1}{\left(k-\frac{1}{2}\right) \pi}\right]= \\
=\frac{\sqrt{2}}{\pi^{2}} \sum_{k=n_{K L}+1}^{\infty} \operatorname{Var}\left[Z_{k} \frac{1}{\left(k-\frac{1}{2}\right)}\right] \leq \frac{\sqrt{2}}{\pi^{2}} \sum_{k=n_{K L}}^{\infty} \operatorname{Var}\left[\frac{Z_{k}}{k}\right]=\frac{\sqrt{2}}{\pi^{2}} \sum_{k=n_{K L}}^{\infty} \frac{1}{k^{2}} \operatorname{Var}\left[Z_{k}\right]= \\
=\frac{\sqrt{2}}{\pi^{2}} \sum_{k=n_{K L}}^{\infty} \frac{1}{k^{2}} \approx \frac{\sqrt{2}}{\pi^{2} n_{K L}}
\end{gathered}
$$

We can scale this approximation to an interval $\left[t_{0}, t_{\max }\right]$ because Brownian motion is self-similar; that is, scaling in time is equivalent to scaling in amplitude ${ }^{\mathrm{b}}$. If we define the following approximation for $t \in[0,1]$ :

$$
\hat{W}(t)=\sqrt{2} \sum_{k=1}^{n_{K L}} Z_{k} \frac{\sin \left(\left(k-\frac{1}{2}\right) \pi t\right)}{\left(k-\frac{1}{2}\right) \pi},
$$

and define $\Delta T=t_{\max }-t_{0}$ then we can build an approximation to $W(t)$ with $t \in\left[t_{0}, t_{\max }\right]$ by:

$$
W(t)=\sqrt{\Delta T} \hat{W}\left(\frac{t-t_{0}}{\Delta T}\right)
$$

and the variance of the amplitude of the error would be scaled by $\Delta T$ as well.

The resulting representation is a smooth approximation of Brownian motion that can be used in direct methods for optimal control, when coupled with the scenario-based formulation in Subsection C.

\footnotetext{
${ }^{\mathrm{b}}$ If $a>0$, then $\sqrt{a} W_{t}$ is equivalent to $W_{a t}$
} 


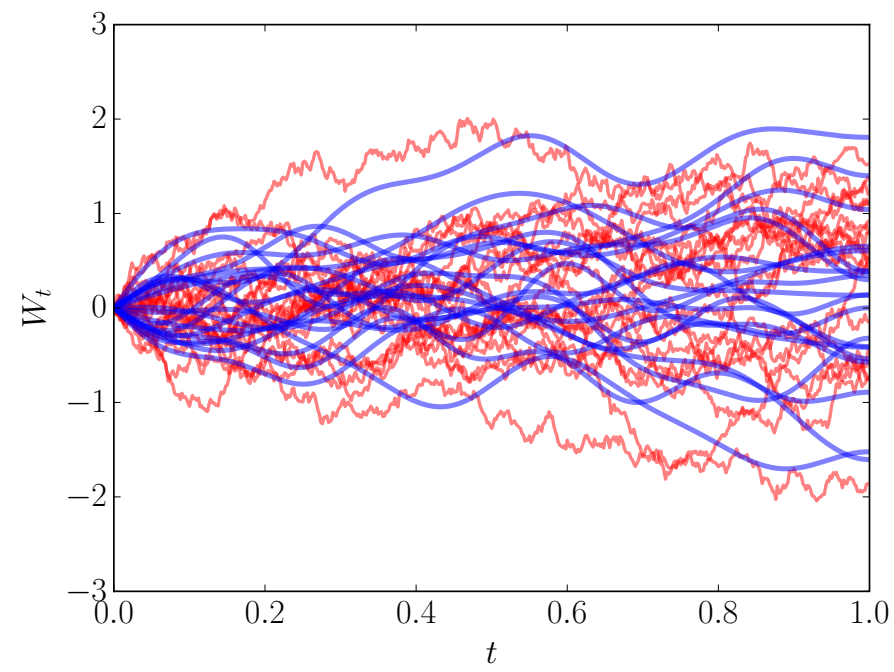

Figure 1. Simulated Brownian motions from Gaussian increments (red) versus Karhunen-Loève approximations of degree 10 (blue)

\section{Storm risk}

Let $p(x, y, t)$ be the time-dependent covering function associated to the storm. If the trajectory of the aircraft is defined by $(x(t), y(t))$, we define the exposure to the storm by the integral:

$$
E=\int_{t_{0}}^{t_{f}} p(x(t), y(t), t) d t
$$

In order to represent the function $p(x, y, t)$, we will represent the uncertain evolution of the storm by a set of $n_{s}$ discrete scenarios. While these scenarios can be generated according to a number of "stochastic quadrature rules" (as discussed in ${ }^{12}$ ), in this work we will restrict ourselves to employing Monte Carlo or quasi-Monte Carlo methods. ${ }^{20}$ In each scenario, we randomly generate $n_{K L}$ normal i.i.d. variables for the Karhunen-Loève approximation and a random orientation $\theta \in U\left(\left[\theta_{\min }, \theta_{\max }\right]\right)$. We will note that the usage of this scenario-based formulation would allow us to easily incorporate different stochastic models of the motion of the storm.

Once we have generated the scenarios, the covering function is approximated by:

$$
p(x, y, t) \approx \tilde{p}(x, y, t)=\frac{1}{n_{s}} \sum_{i=1}^{n_{s}} \mathbf{1}_{\varepsilon_{i}}(x, y, t),
$$

where $\mathbf{1}_{A}$ is the indicator function of set $A$. Let $C^{i}$ be the eccentricity matrix in scenario $i$, computed as in Equation 4 . The position of the center of the storm by $\xi_{i}(t)$ and the position of the aircraft by $q=(x, y)$, the indicator function can be written as:

$$
\mathbf{1}_{\varepsilon_{i}}(x, y, t)=\mathbf{1}_{\mathbb{R}^{+}}\left(1-\sqrt{\left(q-\xi_{i}(t)\right)^{T} C^{i}\left(q-\xi_{i}(t)\right)}\right) .
$$

This equation, however, is not adequate for transcription to a nonlinear programming problem because the step function $\mathbf{1}_{\mathbb{R}^{+}}(\cdot)$ is not continuous. Instead, we replace the indicator function by a sigmoid function:

$$
S_{a}(x)=\frac{1}{2}+\frac{x / a}{2 \sqrt[4]{1+(x / a)^{4}}}
$$


This function is smooth (and, therefore, adequate for usage in a nonlinear programming problem) and approximates the indicator function. The parameter $a>0$ regulates the characteristic length of the transition: a high value produces a smooth and long transition, while a low value produces a sharper transition. Therefore, we approximate the indicator function by:

$$
\mathbf{1}_{\varepsilon_{i}}(x, y, t) \approx S_{a}\left(1-\sqrt{\left(r-\xi_{i}(t)\right)^{T} C^{i}\left(r-\xi_{i}(t)\right)}\right) .
$$

We propose to select a value of $a$ that makes the characteristic length of the smoothing function comparable to the truncation error of the Karhunen-Loève approximation, so that it compensates for this error. From numerical experiments, we estimate the variance of the truncation error to be $0.1 / n_{K L}$, scaled by the time interval $\Delta T=t_{\max }-t_{0}$ under consideration and the trace of the covariance matrix $\tilde{\Sigma}$. The characteristic length of the transition, which we define as $S_{a}^{-1}(0.95)-S_{a}^{-1}(0.05)$, is approximately $2.3 a$ in dimensionless units. We scale it by the geometric mean of the semi-major and semi-minor axes of the ellipse, which we denote as $\sqrt{R R}^{\prime}$. We propose to set $a$ to equate the characteristic length to 4 times the standard deviation of the error, which leads to the rule of thumb:

$$
a \approx 0.56 \sqrt{\frac{\Delta T \operatorname{tr} \tilde{\Sigma}}{n_{K L} R R^{\prime}}}
$$

The exposure $E$ associated to a trajectory can now be modelled in an NLP-friendly way. Note that the exposure does not directly measure the risk of entering the unsafe set; instead, it is an upper bound for this risk. For multiple storms, the total exposure can be created as the sum of the exposure to each storm; the main challenge in this case is determining whether there is any correlation between the stochastic components of the motion of the storms.

\section{Optimal control formulation}

We define the parameter $w_{s}$ that represents the preference for reduced safety risk relative to the preference for earlier arrival to the destination (and the associated fuel savings) if there is no additional rerouting. We can now define the optimal control problem associated to the storm avoidance problem:

$$
\left.\begin{array}{cl}
\operatorname{minimize} & J=t_{f}+w_{s} \int_{t_{0}}^{t_{f}} p(x(t), y(t), t) d t \\
\text { subject to } & \dot{x}=v \sin \chi \\
& \dot{y}=v \cos \chi \\
& \dot{\chi}=(g / v) \tan \psi \\
& |\psi| \leq \pi / 4 \\
& \left(x\left(t_{0}\right), y\left(t_{0}\right), \chi\left(t_{0}\right)\right)=\left(x_{0}, y_{0}, \chi_{0}\right) \\
& \left(x\left(t_{f}\right), y\left(t_{f}\right)\right)=\left(x_{f}, y_{f}\right)
\end{array}\right\}
$$

We use standard direct methods (see, for example, ${ }^{21-23}$ ) for transcription of the resulting optimal control problem into an NLP problem, which can then be solved with an NLP solver. We use IPOPT ${ }^{24}$ as the NLP solver.

In order to ensure that the algorithm converges robustly, we first solve a simpler problem where the heading $\chi$ is taken as a control instead of the bank angle $\psi$ (i.e. without modeling the dynamics of the turn). Then, we take the solution of this problem and use it as an initial guess for the complete problem.

\section{Case study}

We consider a benchmark problem similar to the one described in previous work. ${ }^{9}$ An aircraft is flying in a South-East direction at the latitude-longitude position $\left(29^{\circ},-87^{\circ}\right)$ (near the coast of Florida) at a true airspeed of $220 \mathrm{~m} / \mathrm{s}$. The North American region is projected onto a 2D plane with a Lambert Azimuthal Equal-Area projection centered at $\left(38^{\circ},-98^{\circ}\right)$ as it is done in the CIWS system; ${ }^{15}$ within this region, the 
area of interest in XY coordinates is $\bar{K}=[3600,3800] \times[750,850](\mathrm{km})$. We identify a storm from the VIL forecasts for $01 / 07 / 2009$ having initial position $(x, y)=(3675,776)$, a forecast speed of $[25,19.4] \mathrm{m} / \mathrm{s}$, an eccentricity $M_{k}=[0.0028,0 ; 0,0.0278]$, and a covariance function associated to the stochastic term $\Sigma=\mathbb{I}_{2 \times 2}$ $\left(\mathrm{km}^{2}\right)$.

For the reach-avoid algorithm, we use a sampling time of $\Delta t=1$ minute, an angular velocity for the turning modes of $\Omega=0.3$ radians per minute. We set a disturbance variance of $\Sigma_{w}(1,1)=0.04 \mathrm{~km}^{2}$, $\Sigma_{w}(2,2)=0.04 \mathrm{~km}^{2}, \Sigma_{w}(3,3)=9 \cdot 10^{-6} \operatorname{rad}^{2}$ and $\Sigma_{w}(i, j)=0$ if $i \neq j$. At the specified airspeed, the random variable corresponding to the distance covered by the aircraft in a time step of $\Delta t=1$ minute has a $95 \%$ confidence interval $13.2 \pm 0.4 \mathrm{~km}$. At $\Omega=0.3$ radians per minute, the random variable corresponding to the turning angle covered in the time step $\Delta t=1$ minute has a $95 \%$ confidence interval $0.3 \pm 0.006 \mathrm{rad}$.

The initial position of the aircraft in the XY plane is $(3640,820)$, with an initial heading of $124^{\circ}$. We consider the target set $K=[3742,3768] \times[752,778] \times[-\pi, \pi]$ and the safe set $K_{k}^{\prime}=\bar{K} \backslash \Xi_{k}$. In line with Section E.D, the random closed set is defined as

$$
\Xi_{k}=\varepsilon\left(\xi_{k}, C_{k}\right) \times[-\pi, \pi]
$$

The covering function is approximated on $[3600,3800] \times[750,850]$ using $10^{5}$ Monte Carlo samples over a $101 \times 51$ grid and a stochastic set-valued process with $\xi_{0}=[3675,775]^{T}, \lambda_{k}=[1.5,1.1667]^{T}, M_{k}=$ $[0.0028,0 ; 0,0.0278], \Sigma=\mathbb{I}_{2 \times 2}\left(\mathrm{~km}^{2}\right)$ and $\alpha=\frac{\pi}{6}$. Figure 2 illustrates the covering function associated to the stochastic unsafe set.

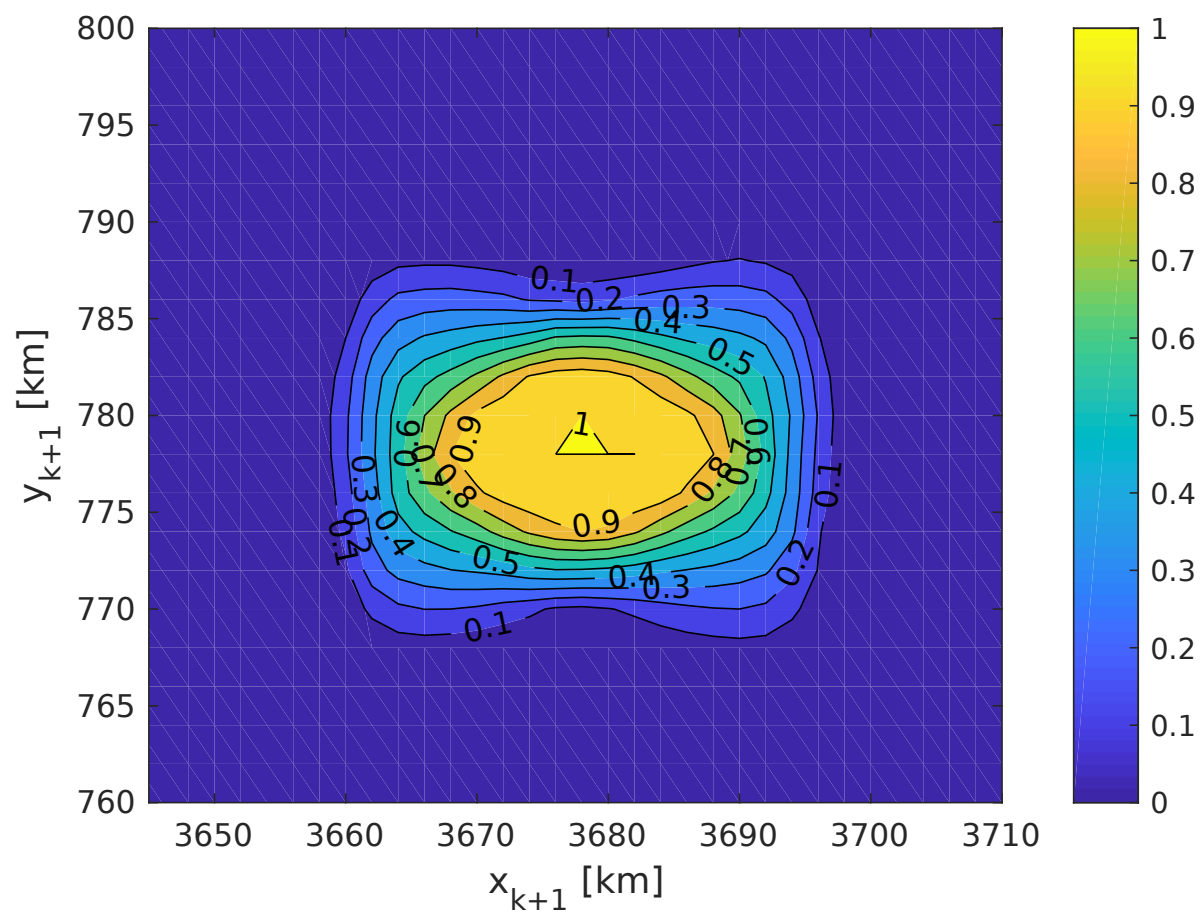

Figure 2. Contour plot of the covering function at time $t=6 \mathrm{~min}$.

According to the method introduced in Section E, we compute the optimal trajectories and associated maximum probabilities of reaching the target set $K$ before intersecting the hazardous weather region $\Xi_{k}$ within $N=30$ time steps, for all starting points in the state space $X^{1} \times X^{2} \times X^{3} \subset \mathbb{R}^{2} \times[-\pi, \pi]$. All numerical computations were performed on a $101 \times 51 \times 42$ grid. 
For the optimal control method, we choose a trapezoidal transcription ${ }^{21}$ with 30 nodes. We further restrict the maximum bank angle to limit the turning rate to $\pi / 2 \mathrm{rad} \cdot \mathrm{min}^{-1}$ for improved passenger comfort and similarity to the reach-avoid formulation. We consider $n_{s}=20$ scenarios, an expansion degree with $n_{K L}=8$ components and a sigmoid smoothing parameter $a=0.07 \mathrm{~km}$, according to the rule of thumb in Section III.C.

\section{Results}

Figure 3 shows the computed trajectories with each method, as well as 30 realizations of the stochastic weather cell process. The represented reach-avoid trajectory is the mean of 1000 Monte-Carlo realizations under the computed optimal policy. It can be seen that the optimal control trajectory reaches the target position faster by tracing a more direct path to both the turning point and the destination point (taking 527 seconds to reach the final position versus 540 of the reach-avoid trajectory), while the reach-avoid framework reroutes only once the probability of ending up in the storm threatens to increase.
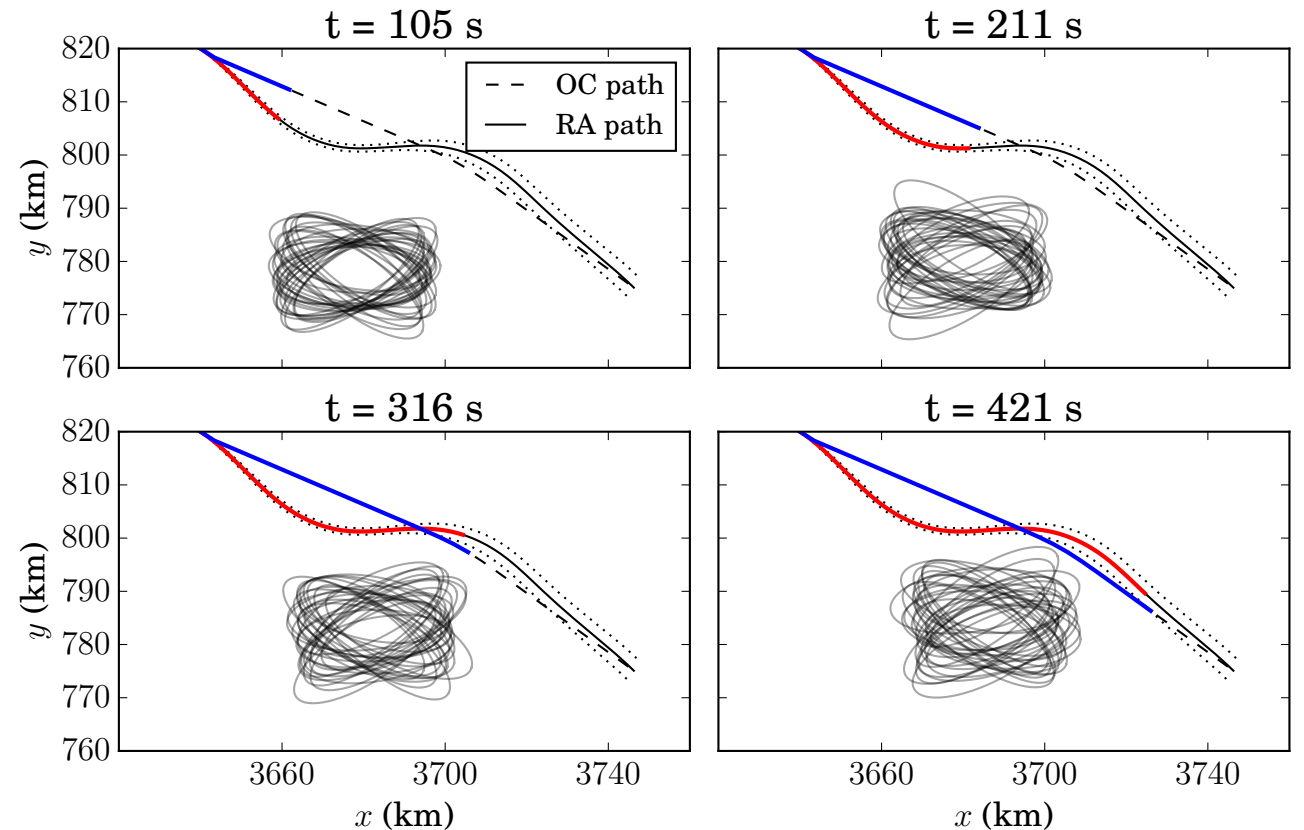

Figure 3. Computed trajectories with the reach-avoid framework (red and solid line, plus $5 \%$ and $95 \%$ quantiles in dotted line) and the optimal control framework (blue and dashed lines). In addition, 30 possible realizations of the stochastic storm process have been plotted.

Figure 4 shows the optimal value function over the state space, that is, the maximum probability of safely reaching $K$ within the given horizon, for an initial heading angle of 2.1124 radians. The level set $V_{0}^{*} / g e q 0.95$, that is, the set of all points in the state space from which trajectories are able to safely reach the target with a probability of at least 95 percent, is shown in Figure 5.

Computational Performance: We have analyzed runtime for both methods. The reach-avoid problem takes 700 seconds to run with a coarse grid of $41 \times 21 \times 32$ points and 480 minutes with the fine grid of $101 \times 51 \times 42$ points, as the curse of dimensionality takes its toll. The optimal control formulation scales better: in the two-step process described in Section D, the first initialization step takes around 20 seconds and the second and final optimization step takes another 20 seconds. Furthermore, we have identified that the main driver of the computational cost of the optimal control problem is the function evaluations associated to the function $p$; this implies that a sizable speed-up can be achieved. Such an improvement could be realized, for example, by synthesizing a computationally cheap approximation of $p$ at a preprocessing step or by evaluating the scenarios in parallel through a technique such as general-purpose GPU computing. 


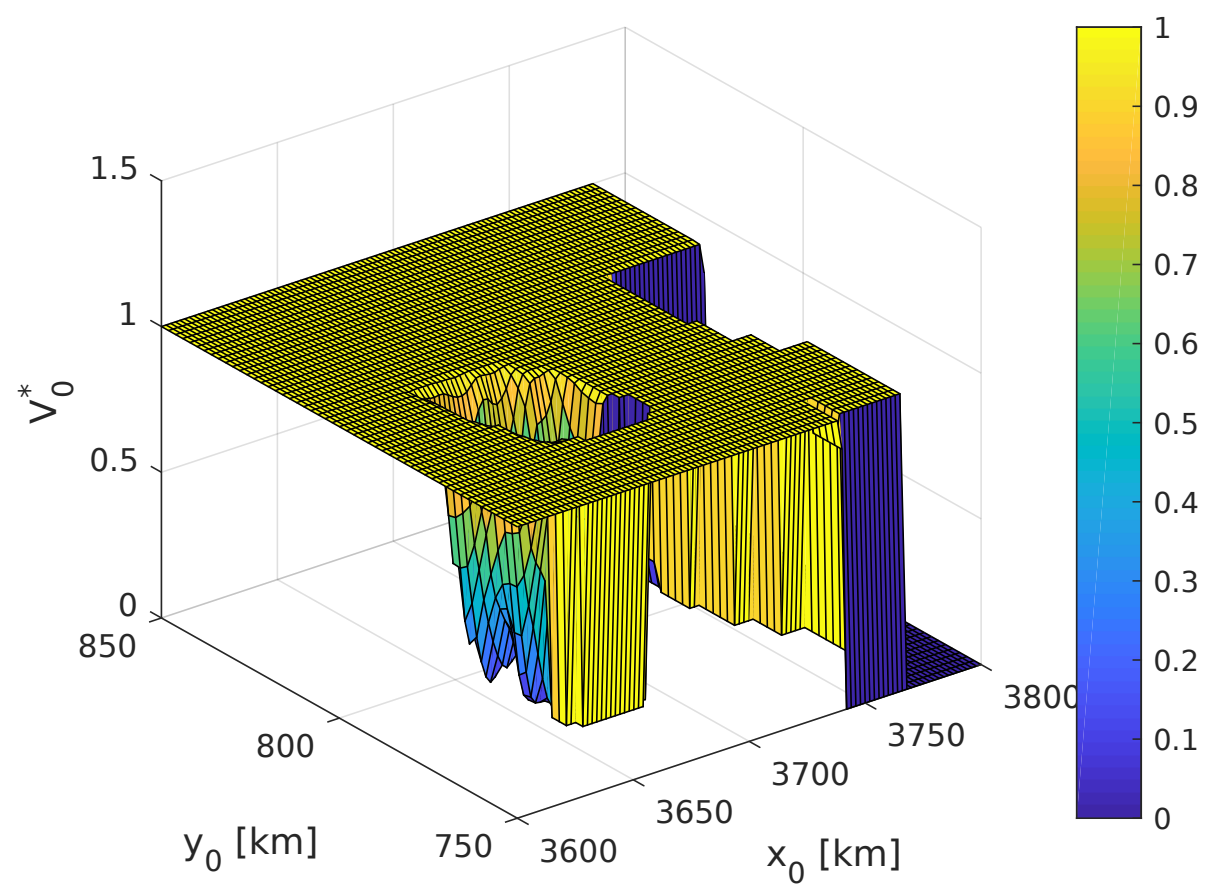

Figure 4. Optimal value function over the state space $\left(\chi_{0}=2.1124\right.$ rad $)$.

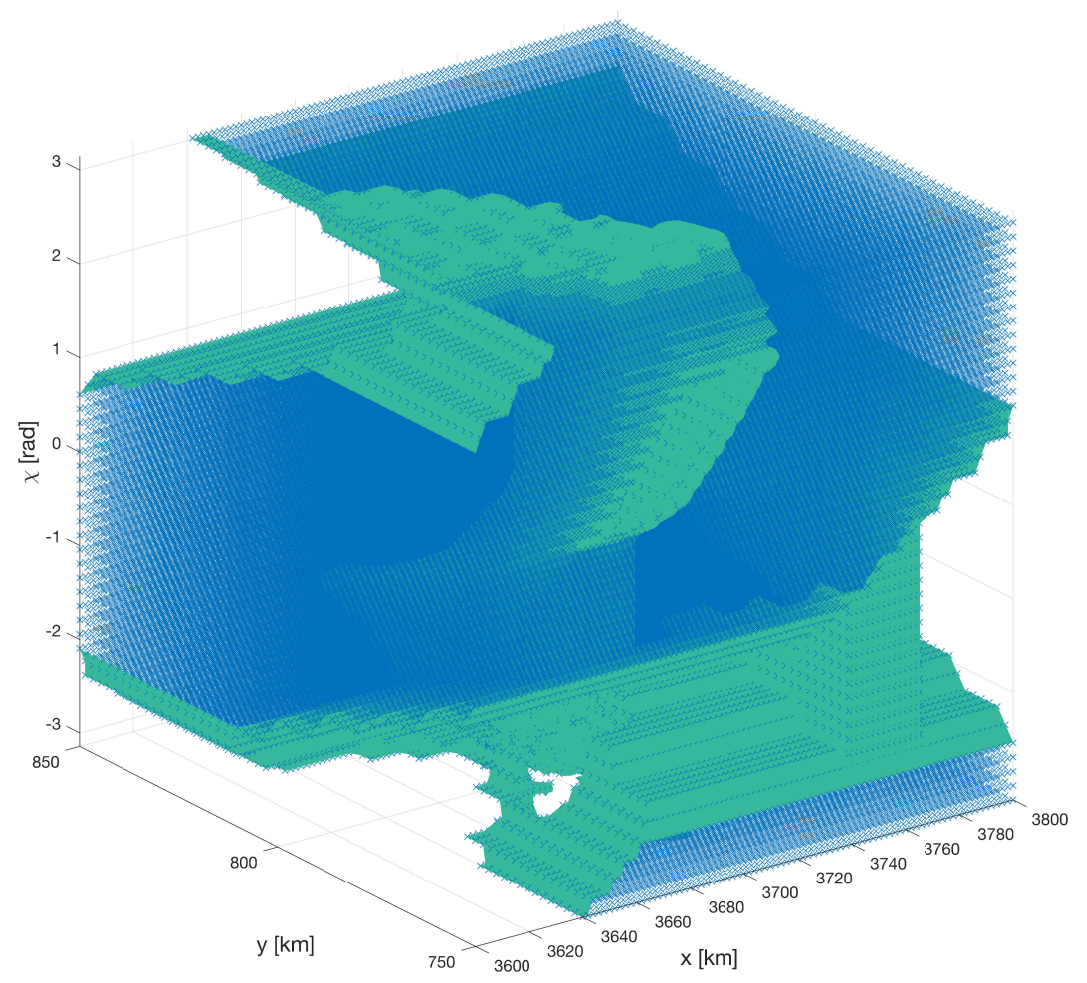

Figure 5. Level set of the value function for $V_{0}^{*} \geq 0.95$. 


\section{Conclusions}

We have presented and evaluated two methodologies for the purpose of automatically generating efficient trajectories that avoid stochastic weather hazards. Both of them are able to calculate safe trajectories, although the levels of efficiency and safety risk for both methodologies exhibit substantial variation. The computational efficiency of the reach-avoid framework is currently low and only useful for analysis. The optimal control methodology performs better in terms of computational tractability and shows some promise for the resolution of the problem at hand in a practical time horizon. Opportunities for improving the computational performance in both methods do exist and we intend to explore them in future work.

We are already working on the automatic application of these approaches with a convective weather forecasting product. This would allow us to test the methodologies on a wider range of cases, as well as to identify and evaluate the stochastic convective cell motion model. Other challenges include dealing with hazards that are not adequately represented as ellipses and incorporating a model for the growth or decay of the storm. More features, inspired by future 4D trajectory management concepts, can be added to the aircraft model, such as variable airspeed profiles. Indeed, we have already incorporated some of these aspects in other work based in optimal control. ${ }^{18}$

\section{Acknowledgments}

This work has been partially supported by project TBO-MET project (https://tbomet-h2020.com/), which has received funding from the SESAR JU under grant agreement No 699294 under European Union's Horizon 2020 research and innovation programme. This work is also partially supported by the Spanish Government through Project entitled Analysis and optimisation of aircraft trajectories under the effects of meteorological uncertainty (TRA2014-58413-C2-2-R). The project has been funded under RD\&I actions of Programa Estatal de Investigación, Desarrollo e Innovación Orientada a los Retos de la Sociedad (call 2014).

\section{References}

${ }^{1}$ National Research Council, Weather Forecasting Accuracy for FAA Traffic Flow Management: A Workshop Report, The National Academies Press, Washington, DC, 2003.

${ }^{2}$ Michalek, D. and Balakrishnan, H., "Building a Stochastic Terminal Airspace Capacity Forecast from Convective Weather Forecasts," Aviation, Range and Aerospace Meteorology Special Symposium on Weather-Air Traffic Management Integration, Phoenix, AZ, 2009.

3 "European ATM Master Plan Edition 2," Tech. rep., SESAR Joint Undertaking, 2012.

${ }^{4}$ McNally, D., Sheth, K., Gong, C., Sterenchuk, M., Sahlman, S., Hinton, S., Lee, C. H., and Shih, F.-T., "Dynamic Weather Routes: Two Years of Operational Testing at American Airlines," Air Traffic Control Quarterly, Vol. 23, No. 1, 2015, pp. $55-81$.

${ }^{5}$ Hauf, T., Sakiew, L., and Sauer, M., "Adverse Weather Diversion Model DIVMET," Journal of Aerospace Operations, Vol. 2, No. 3-4, 2013, pp. 115-133.

${ }^{6}$ Sauer, M., Hauf, T., Sakiew, L., Chan, P. W., Tse, S.-M., and Hupe, P., "On the Identification of Weather Avoidance Routes in the Terminal Maneuvering Area of Hong Kong International Airport," Journal of Zhejiang University SCIENCE A, Vol. 17, No. 3, 2016, pp. 171-185.

${ }^{7}$ Hauf, T., Hupe, P., Sauer, M., Rokitansky, C.-H., Lang, J., Sacher, D., Chan, P. W., and Sakiew, L., "Aircraft Route Forecasting under Adverse Weather Conditions," Meteorologische Zeitschrift, 2017, pp. 189-206.

${ }^{8}$ Sauer, M., Hauf, T., and Forster, C., "Uncertainty Analysis of Thunderstorm Nowcasts for Utilization in Aircraft Routing," Proceedings of the 4th SESAR Innovation Days, 2014.

${ }^{9}$ Summers, S., Kamgarpour, M., Lygeros, J., and Tomlin, C., "A Stochastic Reach-Avoid Problem With Random Obstacles," Proceedings of the 14th international conference on Hybrid systems: computation and control, ACM, 2011, pp. 251-260.

${ }^{10}$ Bertsekas, D. P. and Shreve, S. E., Stochastic Optimal Control: The Discrete-Time Case, Athena Scientific, 2007.

${ }^{11}$ Bellman, R. and Kalaba, R. E., Dynamic Programming and Modern Control Theory, Academic Press New York, 1965.

${ }^{12}$ González Arribas, D., Soler, M., and Sanjurjo Rivo, M., "Wind-Based Robust Trajectory Optimization using Meteorological Ensemble Probabilistic Forecasts," 6th SESAR Innovation Days, Eurocontrol, 2016.

${ }^{13}$ Kamgarpour, M., Dadok, V., and Tomlin, C., "Trajectory Generation for Aircraft Subject to Dynamic Weather Uncertainty," 49th IEEE Conference on Decision and Control (CDC), IEEE, 2010, pp. 2063-2068.

${ }^{14}$ Kamgarpour, M., Wood, T. A., Summers, S., and Lygeros, J., "Control Synthesis for Stochastic Systems given Automata Specifications defined by Stochastic Sets," Hybrid Systems: Computation and Control, 2016, pp. 251-260.

${ }^{15}$ Evans, J. E., Carusone, K., Wolfson, M., Crowe, B., Meyer, D., and Klingle-Wilson, D., "The Corridor Integrated Weather System (CIWS)," 10th Conference on Aviation, Range, and Aerospace Meteorology, 2001.

${ }^{16}$ Nuic, A., "User Manual for the Base of Aircraft Data (BADA) rev 3.11," Tech. Rep. 13/04/16-01, Eurocontrol Experimental Centre, 2010.

${ }^{17}$ Gallo, E., Navarro, F., Nuic, A., and Iagaru, M., "Advanced Aircraft Performance Modeling for ATM: BADA 4.0 Results," 25th Digital Avionics Systems Conference, 2006 IEEE/AIA 
${ }^{18}$ González-Arribas, D., Arnedo, M. S., and Rivo, M. S., "Wind-Optimal Cruise Trajectories using Pseudospectral Methods and Ensemble Probabilistic Forecasts," Proceedings of the 5th International Conference on Application and Theory of Automation in Command and Control Systems, ACM, 2015, pp. 160-167.

${ }^{19} \varnothing \mathrm{ksendal}, \mathrm{B} .$, Stochastic differential equations, Springer, 2003.

${ }^{20}$ Niederreiter, H., Quasi-Monte Carlo Methods, John Wiley \& Sons, Ltd, 2010.

${ }^{21}$ Betts, J. T., Practical methods for optimal control and estimation using nonlinear programming, Vol. 19, Siam, 2010.

${ }^{22}$ Rao, A. V., "A Survey of Numerical Methods for Optimal Control," Advances in the Astronautical Sciences, Vol. 135, No. 1, 2009, pp. 497-528.

${ }^{23}$ Ross, I. M. and Karpenko, M., "A Review of Pseudospectral Optimal Control: from Theory to Flight," Annual Reviews in Control, Vol. 36, No. 2, 2012, pp. 182-197.

${ }^{24}$ Wächter, A. and Biegler, L. T., "On the Implementation of an Interior-Point Filter Line-Search Algorithm for Large-Scale Nonlinear Programming," Mathematical programming, Vol. 106, No. 1, 2006, pp. 25-57. 